\title{
Tracking Gendered Streams ${ }^{i}$
}

\author{
By Maria Eriksson \& Anna Johansson
}

\begin{abstract}
One of the most prominent features of digital music services is the provision of personalized music recommendations that come about through the profiling of users and audiences. Based on a range of "bot experiments," this article investigates if, and how, gendered patterns in music recommendations are provided by the streaming service Spotify. While our experiments did not give any strong indications that Spotify assigns different taste profiles to male and female users, the study showed that male artists were highly overrepresented in Spotify's music recommendations; an issue which we argue prompts users to cite hegemonic masculine norms within the music industries. Although the results should be approached as historically and contextually contingent, we argue that they point to how gender and gendered tastes may be constituted through the interplay between users and algorithmic knowledge-making processes, and how digital content delivery may maintain and challenge gender relations and gendered power differentials within the music industries. Seen through the lens of critical research on software, music and gender performativity, the experiments thus provide insights into how gender is shaped and attributed meaning as it materializes in contemporary music streams.
\end{abstract}

Keywords: gender, music, algorithms, streaming, digital methods

Eriksson, Maria \& Anna Johansson: “Tracking Gendered Streams", Culture Unbound, Volume 9, issue 2, 2017: 163-183. Published by Linköping University Electronic Press: $\underline{\text { http://www.cultureunbound.ep.liu.se }}$ 


\section{Introduction}

Being a user of contemporary online services usually means being "profiled." In broad strokes, the profiling of online users-what is commonly described as a "personalization" of data flows-involves the recording of user behaviors, the approximation of user tastes and preferences, and the delivery of content assumed to fit an individual's needs and wishes. As Mike Featherstone (2006: 592) has argued, similar treatments of data have historically made people "identified and individuated... as whole populations, their bodies and life histories became documented, differentiated, and recorded." Rooted in the practices of nation states, the profiling of populations is increasingly performed by digital media corporations through the treatment of online data. The technical systems that generate this kind of profiling may be understood as "infrastructures of taste formation" with a profound capacity to shape cultural encounters in both descriptive and prescriptive ways (Morris 2012; Beer 2013: 97). Content deliveries that are based on user profiling indicate how the preferences of users are imagined and they also guide the choices users can make. Thus, they may also serve to reproduce certain behaviors.

Working on a project concerned with streaming music, we wanted to explore how the streaming service Spotify may be constitutive of user identities and taste constellations related to gender. Spotify is currently one of the largest providers of digital music, and a company that hosts the music consumption of over 100 million users around the world. Due to its popularity, Spotify is a great example of a new media service that is "central in shaping our everyday lives and in ordering our routine experiences" (Beer 2013: 1). In a previous study, for example, we explored how Spotify evokes normative temporalities, neoliberal subjectivities, and functional approaches to music through its ways of greeting users (Eriksson and Johansson, forthcoming). In this article, our main interest instead lies in the way gender comes to matter in Spotify's music recommendations.

We argue that to fully understand the cultural significance of contemporary online media, scholars in the humanities need to directly engage with the digital systems (algorithms, software assemblages, data flows) that organize media usage. In order to explore whether and how gender is produced through Spotify, we therefore set up a case study that investigated 1) the extent to which users' gender identification had an impact on the provided music recommendations, and 2) the gender ratio of artists recommended to Spotify users. Seen through the lens of previous research on software, music and gender, this provides an understanding of whether algorithmic user profiling and music recommendations are constitutive of gender and gendered tastes, and of how digital content delivery can maintain and challenge gender relations and gendered power differentials within the music industries. 


\section{Algorithms and User Profiling}

On Spotify, algorithmic processes are heavily involved in framing, moving, assembling, and contextualizing music in ways that affect who sees it, and how it is perceived. Over the past few years, studies of online recommendation systems and algorithmic knowledge production have proliferated (Introna \& Nissenbaum 2000; Mackenzie 2006; Amoore \& Piotukh 2016; Kitchin 2017), together with a broader interest in the politics and cultural implications of code and software (Fuller 2003; Berry 2011; Manovich 2013). Algorithms have received attention as "a kind of invisible structural force that plays through into everyday life" (Beer 2013: 69), for instance by providing personalized recommendations of online content. Constituting a new form of power, it has been suggested that the design of algorithms and algorithmic procedures foster certain cultures, ideologies and identities. Mager (2012), for example, has demonstrated how search engines embody an "algorithmic ideology" closely connected to capitalist modes of production, while others have shown how Google perpetuates stereotypes related to race and gender through its deliveries of online content (Olofsson 2015; Baker \& Potts 2013). Because algorithms serve as cultural intermediaries that help content "find us", they also affect how cultural capital is acquired and how taste is shaped (Beer 2013; see also Morris 2015). In this way, algorithms act alongside other cultural intermediaries like record labels, music aggregators, music critics and retailers within the music industries (cf. Drew 2005; Galuszka 2015). Together, such actors perform the task of mediating and creating meaning around music and its audiences. Thus, algorithms are not the only means by which music acquires meaning and fans become profiled, but their involvement in musical processes require further attention.

Currently, critical research on algorithmic filtering systems vary with respect to the extent that algorithms are attributed agency in and of themselves - ranging from studies that theorize algorithms in nonanthropocentric terms; that is, as more-than-human actors which actively shape social life (Parisi 2013; DixonRoman 2016), to studies focusing on how algorithms are developed, deployed and attributed meaning in specific cultural contexts (cf. Seaver 2013; Gillespie 2014). While we take inspiration from the former strand's emphasis on the tangible effects and manifestations of algorithms, we primarily approach algorithmic systems as socio-technical configurations which are inscribed with, and hence performative of, particular world-views when activated by users (cf. Kitchin 2017).

As discussed by Cheney-Lippold (2017), algorithmic ways of organizing content have implications for the construction of gender as well as other social categories. Because of their sorting mechanisms, algorithms both infer and define the meaning of such categories in a process which could be likened to a form of soft biopolitics; that is, a mode of governing bodies and populations. By "deciding 
what it is that the individual encounters, and what it is that they are making a decision about" (Beer 2013: 96), content recommendation algorithms work as prescriptive entities that simultaneously predict user preferences and contribute to the shaping of practices and identities-including gendered forms of existence. Much of this prescriptive work takes place through the profiling of users. As Amanda Modell (2015: 9) has put it, the code and algorithms that power online music deliveries create "positional relations between bodies and sets of music from a seemingly objective standpoint." In this way, "code mediates technoscience and consumer self-knowledge" (ibid.: 4). Therefore, it matters how a service like Spotify imagines and responds to its users.

\section{Software and gender}

Placing our study in the context of current research in the emerging fields of software studies and new media studies, we take the stance that technology is inherently social and political. Feminist studies of technology have emphasized how technology is shaped by the circumstances in which it occurs, and, hence, how "gender relations can be thought of as materialized in technology, and gendered identities and discourses as produced simultaneously with technologies" (Wajcman 2007: 293; for further discussion see also Haraway 1997; Hayles 1999; Sundén 2015). Software, algorithms, and data, we argue, are sociotechnical configurations with discursive as well as material components that are situated in gendered contexts and hence embedded in particular values and gender discourses. According to Bivens and Haimson (2016: 1), design decisions "determine where -in the multiple layers of software-gender appears as a category, how it is materialized within code and activated within software processes, and for what purposes it is deployed." This suggests that digital technologies and infrastructures may be complicit in the reproduction of hegemonic gender relations, but also that they can be put to use for unintended purposes and with unanticipated consequences.

Our study is further informed by the notion of gender performativity (Butler 1990, 2004), which we argue can be fruitfully combined with an understanding of algorithmic systems as performative entities. Following Butler, we take gender to be the material effect of regulatory discourses, and something which only exists to the extent that it is repeatedly enacted in social practice. Binary gendernaturalized notions of masculinity and femininity-are thus seen as a contingent set of positions which are iteratively produced and reproduced through "a stylized repetition of acts" that builds on prescriptive conventions (Butler 1990: 179). By citing existing norms, these can "be exposed as non-natural and nonnecessary when they take place in a context and through a form of embodying that defies normative expectations" (Butler 2004: 218). ${ }^{1}$ 
While Butler's notion of gender performativity mainly focuses on how gender is brought into existence by linguistic and embodied practices, she claims that performative power is also exercised through "organizations of human and nonhuman networks, including technology" (Butler 2010: 150). In the specific context of gender and software, a similar line of thinking has also inspired studies of how programmed configurations of gender materialize on social media services, and how gendered technologies are mutually shaped by users and software designers (Bivens \& Haimson 2016). In a study of design decisions related to gender on Facebook, for example, Bivens (2015: 2) shows how code and software can be seen as producing "the conditions for gendered existence" by normalizing a binary logic. Algorithmic music recommendations, we suggest, represent another potentially illustrative example of how software comes to regulate social life.

\section{Gender and music}

Importantly, algorithmic music recommendations are tightly interwoven with the music industries at large, where gender is of major significance. The persistent male domination in the music industries has been noted by several scholars over the years, hence acknowledging the marginalization of women in music production and the ways in which gender conventions and ideologies affect music practices (Frith \& McRobbie 1991; Whiteley 2013; Gavanas \& Reitsamer 2013). For instance, it has been noted how recording studios, tour buses and guitar shops are constructed as masculine contexts (Bayton 2013; Leonard 2007) and how technological mastery is strongly associated with male expertise (Gavanas \& Reitsamer 2013). As a consequence, female musicians are often designated as exceptions, thus normalizing the male performer (Leonard 2007; Gadir 2016).

Prescriptive ideologies of masculinity and femininity are also "bound up with particular musical styles" (Whiteley 2013: xix), and gender ideologies inform the valuation of different music genres for performers and fans alike. Here, the rock/ pop binary is arguably the most well-cited: scholars have problematized the ways in which pop is typically attributed feminine characteristics and a mainstream, commercial orientation, whereas rock music is seen as masculine, authentic and of higher value - thus reproducing the marginalization of women in rock (Railton 2001). Similarly, gender relations are played out and naturalized in relation to other genres, manifested for example in the the co-construction of white masculinity and indie rock (Bannister 2006), the devaluation of women and gay rappers (Jeffries 2011; Berggren 2013), and male dominance in DJ culture (Gadir 2016). Typically, such studies point to the interconnectedness of gender, race, class and sexuality in relation to different music styles.

At the same time, feminist scholars have noted how patterns of domination 
and exclusion in popular music are negotiated and subverted, and thus how music can also act as a vehicle for transformation of gendered and sexual subjectivities (Pough 2004; Whiteley \& Rycenga 2013). It has been suggested that digital technology, especially social media, enable women's self-production of music (Choi 2016), but also that digitalization perpetuates the view of technologies as domains of masculinity and male expertise, in the context of music production (Gavanas \& Reitsamer 2013) as well as consumption (Werner \& Johansson 2016). However, while a number of studies have explored the impact of emerging streaming technologies on music distribution and music practices (Morris 2015; Kjus 2016; Maasø 2017), few have specifically addressed the significance of gender in these processes. Exceptions are for example Werner and Johansson $(2016,178)$, who discuss how "music and technology emerge as gendered in talk about contemporary online music use." The present study contributes to the field by focusing on the embeddedness of gender discourses in media technologies themselves, and by suggesting innovative methods for the study of gender, music and digital technology.

\section{Bot Methods}

For the purpose of this study, we arranged an experiment that explored similarities and differences in music recommended to Spotify users registered as male and female. ${ }^{2}$ The experiment was carried out with the help of system developers Roger Mähler and Johan Von Boer at Humlab, Umeå University. Grounded in digital methods and the use of software affordances as research tools (Rogers 2013), the experiment involved the creation of programmed informants-essentially coded scripts, or bots-that were instructed to behave like ordinary users. ${ }^{3}$ In taking such an approach, we wanted to directly engage with Spotify's software, rather than studying its dynamics as they are mediated by its company representatives in traditional industry interviews or public documents. In alignment with Evelyn Ruppert, John Law and Mike Savage (2013), we argue that scholars who research digital technologies need to get their hands dirty and explore the affordances of digital devices and how they collect, store, transmit, sample, and forge social relations. By experimentally engaging with digital technologies and testing the boundaries of what can be known about Spotify's recommendation systems from the outside, we hope to contribute to such an emerging conversation.

Currently, little is known about how Spotify's recommendation algorithms operate in relation to gender, although clues might be drawn from blog posts such as "Gender Specific Listening", written in 2014 by Paul Lamere, director of the developer platform for the Spotify-owned company The Echo Nest. The Echo Nest has helped manage Spotify's music recommendations since many years and in his 
text, Lamere argues that gender is one of the key demographic variables that can say something about a user's taste in music. Based on the analysis of historical user data, Lamere explains that identifying and eliminating artists that are "gender skewed" is one of the prime strategies by which gender might be used to modify music recommendations. His argument reveals that notions of "gender specific music tastes" exist among software developers, but the text does not confirm that gender-adapted recommendation schemes are actually put to use in the Spotify service. ${ }^{4}$ This is what we set out to investigate in our research.

In the experiment, we began by first registering 288 new Spotify accounts. ${ }^{5}$ These accounts had the exact same settings (address, date of birth, home address, privacy settings etc), but half of the users were registered as male and half as female. At the time, male/female were the only gender options available upon registration to the service (In September 2016, Spotify began to roll out the possibility of registering a third gender category in select countries - an issue we will return to in our discussion. $)^{6}$ The 288 accounts were then divided into four groups, and the bots in each group were instructed to listen to music from one of the genres rock, gospel, rnb/hiphop, and dance/electronic music. The genres were borrowed from Billboard's global hit lists at the time of the study, and the users were instructed to listen to the ten most popular songs on Billboard's top 100 hit list within each genre. In total, 72 accounts (half male and half female) were assigned to each music genre, and each user account was connected to a programmed script that 'mimicked' the behavior of ordinary Spotify users. This included signing in to the service, playing 10 selected songs, and signing out again, according to the following schedule:

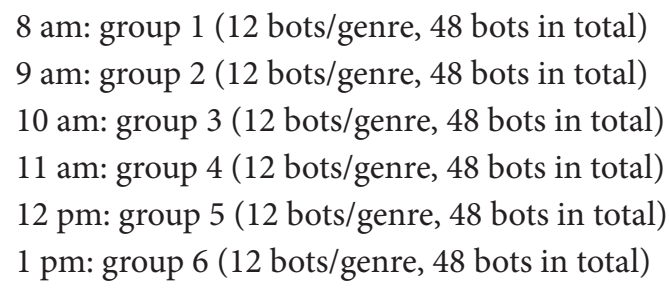

After each session, a script documented the music recommendations provided to the users in Spotify's Discover section. Because of their programmed nature, the bot users never made any mistakes (such as clicking on the wrong link, or accidentally skipping a song), which is very different from human users. We do not know if the Spotify client could sense their programmed nature, but we received no indications that it did. Using randomized behavioral patterns for the bots could have decreased their "robotic" conduct, but it also would have made comparisons between users much more difficult. Hence, we decided to stick to a 
controlled experimental setup. In total, data was collected once per day for each user, during four days between June 18 and 22, 2016. The data was collected using 10 virtual Windows computers and was saved in the shape of screenshots and html data. We conducted the analysis using Microsoft Excel and Google Spreadsheets.

As a whole, our experiment bore some similarity to reverse engineering, a strategy that aims to figure out how technology works by back-tracking its outputs. By studying what kind of music recommendations Spotify delivered to predesigned users, we were hoping to understand more about patterns in the system's outputs. It has been pointed out, however, that reverse engineering comes with many problems, such as the inability to say something about the cultural work that lies behind the system, and approaching the digital sphere as something stable whose inner workings can be fully discerned (Seaver 2014, see also Introduction and Snickars in this issue). We want to stress that although our study is partly informed by reverse engineering methodologies, our primary interest was not to uncover any presumed 'secrets' in Spotify's music recommendations. Therefore, our analytical approach focuses less on how recommendations function and why, and more on what they do in the world.

Knowing that there would always remain blank spots and inconsistencies in the data (since digital technologies are inherently slippery and mutable), we also approached the Spotify service as a "black box" in the classic cybernetic sense of the term (see Pickering 2011: 21). ${ }^{7}$ This involves seeing black boxes as inherently ungraspable and ubiquitous, rather than as technical systems that might become fully transparent to our understanding. In this way, our approach to Spotify's music recommendation system may be described as a process of "tinkering", rather than a strict and rule-bound scientific experimentation. In a "tinkering" spirit, the process of arriving at the results in this article were also far from linear and involved many detours, adjustments, and reconsiderations. ${ }^{8}$

\section{Tracking Gendered Streams: Results from a Bot Experiment}

The focus of our analysis has been the supposedly personalized music recommendations delivered as "Top Recommendations for You" in Spotify's Discover section. " "Top Recommendations for You" is the first content category that users meet when browsing this section, and it can therefore be seen as particularly significant in terms of positioning users and producing meaning around music. The analysis was limited to recommended artists, which means that we did not consider whether users had been recommended different songs or albums by the same artist. Furthermore, since data was collected once per day and Spotify's music recommendations did not update on a daily basis, many artists appeared multiple times for the same user. Such duplicates were removed from the 
data set. In sum, our analysis was based on 492 different artist recommendations that were displayed to our bots during the course of the experiment.

In the first part of the analysis, we explored the extent to which male and female registered users within each music genre had been given the same artist recommendations. This would tell us whether Spotify's recommendation algorithms seemed to assume that our male and female bots had the same taste in music or not. If male and female bots in each genre were given identical recommendations, it could be inferred that the recommendation algorithms had not treated them differently. If the bots were given non-matching music recommendations, however, we would be able to say that within the scope of this particular experiment, Spotify's music recommendation system seemed to respond to the registered gender of the bots.

The results of this analysis showed that 86 percent of the $\mathrm{rnb} / \mathrm{hiphop}$ bots, 93 percent of the rock bots, 93 percent of the gospel bots, and 78 percent of the dance/electronic bots had largely been recommended music by the same artists, irrespective of their registered gender. The remaining bots had been given very different sets of recommendations. We call such bots "outliers," and the extent to which these bots were male or female differed marginally between the music genres. More specifically, four female and six male rnb/hiphop bots, one female and four male rock bots, three female and two male gospel bots, and nine female and seven male dance/electronic bots were defined as outliers. In other words, there were small indications that our male and female registered bots had been differently targeted as outliers, but it would be precarious to draw any conclusions from this result since the total number of outliers was so small. Interestingly, however, the dance/electronic bots received an overall higher percentage of outlier recommendations than the bots in the other genres.

On the other hand, the analysis showed that a few specific artists were recommended to slightly more male than female registered users, or vice versa. Such seemingly gender skewed recommendations were most common in the rock genre. Here, for instance, 19 of the female bots, but only 12 of the male bots had been recommended the "poppy, jittery, upbeat, math rock/post-punk sound" of the British all-male band Foals. ${ }^{10}$ Similarly, the all-male band The Neighborhood, mixing "atmospheric indie rock, electronica, and hip-hop beats with melodic $\mathrm{R} \& \mathrm{~B}$-inflected vocals", was recommended to 32 male bots, as compared to 27 female. ${ }^{11}$ We found several examples of slightly gender skewed artists in the rnb/ hiphop and dance/electronic genres too, ${ }^{12}$ but not in the gospel genre.

In the second part of the analysis we explored the gender ratio among the musicians recommended to our bots. For this purpose, we tagged every artist recommendation according to the gender presentation of the artist. Our reading of gender was based on one or several of the following elements: the pronoun 
used in texts about the musician(s), the name of the artist or band members (artist name or personal name), and/or photographs of the artist or band. If a duo or band consisted of both male and female artists, they were tagged as "mixed."13 We did not find any musician who explicitly positioned themselves outside the gender binary. By using the criteria above, we were able to define the gender of 485 (or 99 percent) of the unique artist recommendations given to our bots. ${ }^{14}$

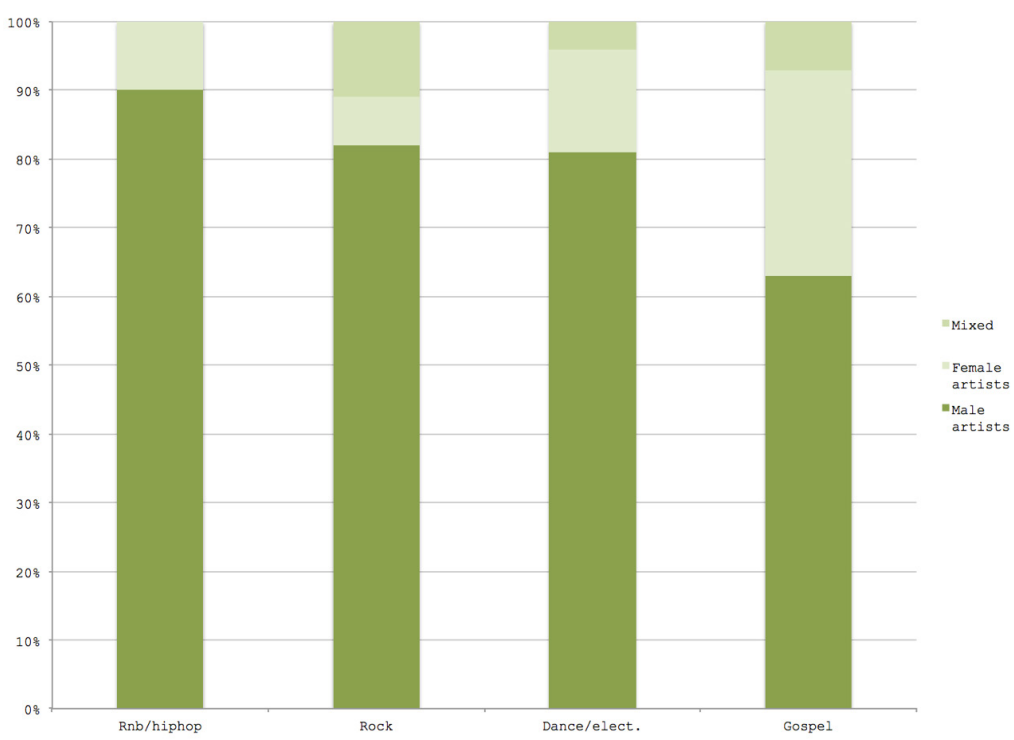

Table 1. Percentage of recommendations for male artists, female artists, or mixed bands (irrespective of bot gender). The percentages correspond to the following number of artist recommendations: Rnb/hiphop (1730), Rock (2246), Dance/Electronic (1682), Gospel (1644).

Out of these 485 artists and bands, 386 (or 80 percent) were identified as male, and 73 (or 15 percent) were identified as female. 24 (or 5 percent) were tagged as mixed duos or groups. Thus, male artists were highly overrepresented in Spotify's music recommendations during the course of the experiment. Since Spotify does not publicly announce (or perhaps even register) the self-attributed gender of their artists, we do not know if these figures are representative of the overall gender proportions of available artists on the service. But in any case, our figures revealed that a vast majority of the artists recommended during this particular experiment performed as male. When investigating whether the registered gender of our bots was related to the gender presentation of the artists recommended to them, we found that although male and female users in each genre were given some nonmatching artist recommendations, the proportions of male artists, female artists and mixed bands were almost identical. 
Table 1 shows the percentage of recommendations for male artists, female artists, and bands with both males and females within each music genre when duplicates between bots were included-figures which, again, were almost identical when the data was broken down into male and female users. Neither did we find any siginificant differences between outliers and other bots. As the table demonstrates, rnb/hiphop was the genre with the highest dominance of male artist recommendations ( 90 percent), followed by rock ( 82 percent), dance/ electronic ( 81 percent), and gospel (63 percent). The rnb/hiphop users were the only group of bots that did not receive any recommendations concerning bands with both male and female artists.

The gospel bots received the highest percentage of recommendations of female artists (30 percent), as well as the second largest percentage of bands with both male and female musicians. The rock and dance/electronic bots were recommended almost the same share of male artists and bands ( 82 percent and 81 percent respectively). However, the rock bots had a higher percentage of mixed bands in their recommendations ( 7 percent female artists, and 11 percent mixed duos/groups), while the dance/electronic bots received more female artist recommendations ( 15 percent), than mixed groups (4 percent).

To summarize, our analysis indicated that overall, Spotify's music recommendation system had not treated our male and female bots differently. In fact, between 78 and 93 percent of the male and female bots in each music genre were given nearly identical recommendations. The remaining percentages were made up of users that had been given a large number of outlier recommendations. While the tendency for users to be positioned either as 'outliers' or as adhering to the genre norm is an interesting finding in itself (and a topic of discussion which we will get back to shortly), we could not find any significant indications that this was related to the registered gender of the users.

Further, our analysis did not indicate that Spotify's music recommendation algorithms assumed our male and female registered bots to have different preferences regarding the gender of artists. Instead, male and female bots within each music genre were largely recommended the same percentage of male artists, female artists and mixed duos or bands. However, the analysis did show that Spotify's music recommendations were heavily geared towards recommending music by male artists to all users during the experiment. If our bots would have continued to listen to the music they were recommended, between 63 and 90 percent of their musical intake would have come from male artists (depending on genre). In the genre with the least female artists (rock), only 7 percent of the recommendations concerned female artists. 


\section{Discussion: Gendering Music Streams}

To some extent, the results of our experiment support the notion that popular music is a gendered phenomenon. However, the gendering of Spotify users and the tracking of gendered streams have shown to be neither straightforward nor unambiguous. Jumping back to the initial stages of our experiment, the requirement to register gender-male or female-when signing up for the service was itself a precondition for this study. The mandatoriness of taking up a gendered position, together with the compulsory self-identification within the confines of binary gender, can be seen as an indication that user profiling based on gender was considered vital to the functioning of the software and its recommendation system, or that such profiling was central to the company's monetization strategies (Bivens 2015). In either case, following Butler (2004), the mandatory gender registration illustrates how identification as either male or female is a performative act, necessary for the production of intelligible subjectivity in user interaction with the service. An obvious effect of the requirement to take up such narrow "menudriven identities" (Nakamura 2002) is that people identifying outside the gender binary have to either abstain from using the service, or choose to misrepresent themselves-issues which, for several years, have spurred criticism in the Spotify user community. ${ }^{15}$

During the fall of 2016, Spotify opened up for additional forms of selfidentification by adding a third option to their mandatory gender field, now consisting of "male", "female", and "nonbinary". This was in line with developments seen in other services, such as Facebook, where the launch of custom gender options in 2014 gained much media attention (Bivens \& Haimson 2016). However, as Bivens (2015: 6) demonstrate in her study of the Facebook API, "deep in the database, users who select custom gender options are re-coded-without their knowledge-back into a binary/other classification system that is almost identical to the original 2004 database storage programming". This, Bivens argues, is a way of simultaneously serving users' need for genderqueer identifications, and offering advertising clients "a more marketable and 'authentic' (yet, paradoxically, misrepresented) data set” (Bivens 2015: 7). Because Spotify's third gender option was introduced after the finalization of our data collection, we have not been able to interrogate its materialization in code or its effects on music recommendations; these will be important questions for future studies. We note, however, that Spotify first rolled out the nonbinary feature in a few select countries (including Sweden, Australia, UK and the US), ${ }^{16}$ with countries such as Brazil, Canada, France, Germany, Italy, Japan and Mexico still being limited to the binary options at the time of writing this article. Furthermore, gender identification (within or outside the binary) is still compulsory, which suggests that gender data is even now considered critical to the service's profiling of users. 
While the compulsory, menu-driven identification brings gendered subjects into existence on the service, it is not immediately clear whether and how gender is continuously enacted in the following interplay between users and the algorithmic system. Our case study demonstrated that, overall, the bots in each music genre had received the same music recommendations, regardless of their self-attributed gender. In selected cases, we detected minor differences in terms of the extent to which our male and female bots were positioned as outliers, and in the extent to which our male and female bots had been targeted with specific artist recommendations. However, these differences were very small, and it cannot be known whether they are actually a consequence of algorithms responding to the initial gender presentation of our bots, randomness, or results of beta-testing a new system.

In contrast to the mandatoriness of gender identification at registration, this absence of gender-specific recommendations illustrates that software solutions may carry the potential to move beyond essentializing notions of identity. As John Cheney-Lippold (2011) notes, there lies a progressive potential in how algorithmic contexts construct identity categories such as 'male' or 'female' as neither fully self-selected nor "determined by one's genitalia or even physical appearance" (ibid: 165). Instead, categories are flexible and fluid, continuously inferred upon individuals based on their practices and doings, as compared to other individuals' practices and doings (cf. Bivens \& Haimson 2016). In our case, despite their selfattributed gender, most bots in each genre seemed to be constituted as similar because they all listened to the same music in the same way.

Such constant feedback loops of user behaviors and algorithmic content filterings can be said to accentuate the performative character of the service as well as the ways in which identity categories become open to negotiation (Cheney-Lippold 2017; Kitchin 2017). Because users are requested to engage in continuous acts of music selection and deselection, they can also challenge and transform any normative expectations that might come with these requests (cf. Butler 2004). This may be done through intentional acts of resistance, such as misrepresenting one's gender when signing up for the service. It may also happen when a user intentionally or unintentionally ignores the prescribed content. In either case, Spotify's system for delivering music recommendations is not a closed entity which inescapably steers user behavior, but a system that is open to acts of contestation at the front-end, as well as to development and transformations at the back-end. In other words, algorithms "are never fixed in nature, but are emergent and constantly unfolding" (Kitchin 2017: 21).

While the performative and citational nature of recommendation systems may allow us to move beyond essentializing ideas of binary gender, such systems might also-as Cheney-Lippold (2017) has noted-lead to new forms of dynamic, 
statistical stereotyping based on behavior rather than demographic categories. This was indicated by the fact that bots listening to different genres also received different recommendations, although our study did not give detailed information about how this dynamic categorization worked. However, every user will in some sense always feed conventionally gendered data into the Spotify system, because they can only make themselves known to the service as conventionally gendered subjects (male, female, or-in select cases-nonbinary).

One additional and unexpected result of our analysis was that some users were constructed as outliers in terms of their music recommendations. While the meaning of such odd user profiles could arguably be interpreted in different ways, we suggest that outlier recommendations position certain users as less mainstream, and more niche and exploratory than others in their music taste. We could find no clear signs that the users' self-attributed gender coincided with outlier status, but we did notice differences between the genres. Dance/electronic was the genre with most outlier bots (22 percent), as compared to rnb/hiphop (14 percent), and rock/gospel, where only 7 percent of the bots had been treated as outliers. This suggests that dance/electronic fans are more often treated as exploratory and adventurous in their music taste, as compared to their rock and gospel counterparts. Given that musical connoisseurship, expertise and agency are characteristics that have frequently been associated with masculinity (eg. Straw 2013; Werner and Johansson 2016: 187), such differences between genres might be said to reinforce the gendering of music styles, possibly constructing dance/ electronic-and to some extent $\mathrm{rnb} /$ hiphop music-as more niche, exploratory and hence masculine genres than rock and gospel. This is in contrast to some traditional co-constructions of gender and music style, such as the positioning of rock music as masculine (Railton 2001; Bannister 2006; Whiteley 2013).

The most significant result of the study concerned the extent to which our bots had been recommended music by either male or female artists. ${ }^{17}$ Our study revealed an overwhelming majority of male artist recommendations in all four music genres. This comes as no surprise, given that the music industry has long been understood as a male dominated domain and, hence, a field in which other gender positions are marginalized (Leonard 2007; Cohen 2013). Still, we find the results remarkable. While there were exceptions to the construction of male-asnorm among musicians, male-defined artists were indisputably privileged during the course of our experiment. It should again be noted that music recommendations provided by algorithms operating under the hood of the Spotify client are created and developed in a larger context (cf. Seaver 2013; Kitchin 2017) and also work together with a range of other cultural intermediaries such as record labels, music aggregators, and music critics. Spotify is thus not an isolated actor in generating gendered streams, but the service did appear to contribute to the construction 
of music production as a domain of masculinity. Indeed, the privileging of male artists could be read as producing this streaming service as a masculine context in itself.

Such gendering of music in general, and of the Spotify service in particular, has representational and material effects for both fans and musicians, which points to another aspect of the performative power of the recommendation system. At a symbolic level, the gender representation in artist recommendations encourages specific ways of defining and recognizing musical success. For instance, few female artist recommendations imply fewer opportunities for imagining music talent as a property of femininity-for fans as well as for artists. Notably, the skewed representation might also have material effects in the sense that male artists receive greater financial compensation. Spotify is uniquely positioned to ensure more plays for artists through selective exposure and promotion. But as our study demonstrates; this curatorial authority was deployed in ways that most likely maintained male material privileges in the music industries. Both a consequence and a cause, our bots were urged to financially support and sustain the fame of male musicians. Thus, they were requested to take part in a particular construction of binary gender as well as its power differentials.

\section{Concluding Remarks}

The digital methods used in this experiment has enabled an analysis of Spotify's recommendation system during specific sampled circumstances, which brings with it certain limitations. Most notably, the algorithmic structures behind music recommendations continuously change due to developer decisions as well as to the feedback loops that adjust the system's outputs (see e.g. Cheney-Lippold 2011, Seaver 2014). This means that our study is not necessarily replicable or generalizable, as the software system we were engaging with in June 2016 was most likely very different from today. Nevertheless, our study has provided insights into the ways in which the interplay between Spotify's recommendation system and its users is a performative process through which user identities are continuously produced and enacted. Importantly, however, such performative processes do not necessarily involve the construction or reinforcement of gendered music preferences. As demonstrated in this article, Spotify did not appear to infer gendered taste profiles on our bot users. In a majority of the cases, our male and female bots were given identical music recommendations. What the study did show, however, was that Spotify had displayed an equal tendency to recommend male artists to both our male and female bots. In extension, this implies that our users were prompted to take part in the iterative co-production of male-as-norm in the music industries, thereby also reproducing hegemonic gender conventions of masculine artistry and fame. 
As a last remark, we want to point out that music recommendations only represent one of the ways in which gender matters on Spotify, and other aspectssuch as advertising strategies-may be even more important for how gender materializes on the service. As Bivens (2015) has noted, the mandatoriness of gender registration indicates that gender profiling is essential, either to the functioning of the software or to the service's monetization strategies. Because gender profiling did not seem to have an immediate impact on music recommendations, one might speculate whether it is instead criticial for advertising purposes.

Moreover, gender is not the only user-provided identification that is requested upon registration and thus have the potential to affect music recommendations. Other identity markers such as age, cell-phone type, or country of residence may be worthy of attention and can possibly intersect with gender performances in complex ways. In future research, then, we believe that key insights could be gained by analysing gender alongside other social categories made relevant in algorithmic user profiling. Relatedly, ethnographic studies of software design processes and the rationales behind developers' design decisions (e.g. Seaver 2014), as well as research on the perceptions and practices of streaming service audiences (e.g. Nylund Hagen 2015; Werner \& Johansson 2016), provide important contextualizations for our study. However, while this type of research contributes to a broader understanding of the gendered dimensions of Spotify's music recommendations, we suggest that scholars in the humanities and social sciences also need to experiment with new ways of engaging with and knowing about digital services. Bot methods might—as we have hopefully shown — provide one such opportunity.

Maria Eriksson is a $\mathrm{PhD}$ candidate in Media and Communication Studies and an affiliated researcher at Humlab, Umeå University. Her background is in Social Anthropology, with a special interest geared towards digital and economic anthropology. E-mail: maria.c.eriksson@umu.se

Anna Johansson is an Associate Senior Lecturer in Ethnology at HUMlab, Umeå University. After receiving her $\mathrm{PhD}$ in 2010, her research has focused on social media and gender, online health activism and processes of politicization, and the body in digital culture. Major research interests are digital ethnography and qualitative methods. E-mail: anna.johansson@umu.se 


\section{Notes}

i Some of the digital methods used in this article are non-compliant with Spotify's Terms of Service (ToS). The data collection has ended and did not involve any user data. With the public and academic interest in mind, we appreciate Spotify's forbearance with any trespassings of ToS that our data collection involved.

${ }^{1}$ While it is important to point out that heteronormativity (or the entwinement of sex, gender and desire) is constitutional for binary gender in Butler's work, our study focuses mainly on the gender dimension.

${ }^{2}$ For a similar methodological approach, see for example Boshmaf et al (2011), Eriksson and Johansson forthcoming, and Snickars this issue. Also see the work of Christian Sandvig et. al, available at http:/auditingalgorithms.science/ (accessed 10/10/2017).

${ }^{3}$ For a similar approach to experimental research in the humanities and social sciences, see for example Nigel Thrift's (2008: 12) discussions on 'playful' experimental methods.

${ }^{4}$ Arguments which are similar to Lamere's have also re-surfaced on Spotify's own blog, see https://insights.spotify.com/se/2014/02/10/men-and-women-as-music-fans/ (accessed 27/10/2016).

${ }^{5}$ Limiting the study to 288 bots primarily had to do with hardware restrictions. More users would require more processing power than we had access to.

${ }^{6}$ The gender neutral option is discussed in this post: https://community.spotify. com/t5/Implemented-Ideas/Make-a-gender-neutral-option-for-profile-sign-up/

idi-p/482938 (accessed 11/04/2017). Because our data collection was finalized in June 2016, however, we were not able to include this third gender category in our set-up. This calls for future research and demonstrates the precariousness in studying digital services that are constantly subjected to updates and modifications - an issue which we will return to in the final discussion.

7The concept of the "black box" has been used in many ways - not least within the field of Actor Network Theory, and in the works of Bruno Latour. Here, however, we solely wish to denote the cybernetic use of the term as laid out by Pickering (2011). ${ }^{8}$ Before the data presented in this article was collected, we had done two (less successful) pre-studies. Initially, we had troubles establishing a stable system of data collection. During the second pre-study for instance, a majority of the bots were not given any music recommendations at all, and we were unable to find out exactly why. This shows how difficult it is to engage with algorithmic systems whose operational logics are hidden.

${ }^{9}$ The remaining categories of music recommendations that were not included in the analysis include "Discover Weekly," "New Releases For You," and "Because You Listened to XXX...”

${ }^{10}$ See https://open.spotify.com/artist/6FQqZYVfTNQ1pCqfkwVFEa (accessed 09/05/2017) 
${ }^{11}$ See Https://open.spotify.com/artist/77SW9BnxLY8rJ0RciFqkHh/about (accessed 10/10/2017).

${ }^{12}$ For example, in the rnb/hiphop group, none of the female bots, but six of the male $\mathrm{rnb} /$ hiphop bots had been recommended music by the artist J.R., and in the dance/ electronic group, six female and only one male bot had been recommended music by Robin Thicke, one of the most "charismatic, flashy and commercially successful R\&B acts of the 2000s and 2010s", according to Spotify. See https://open.spotify. com/artist/0ZrpamOxcZybMHGg1AYtHP (accessed 14/05/2017)

${ }^{13}$ While we recognize that this is a problematic undertaking and that there might be cases where our assumptions might not match the self-identification of artists, we believe that pronouns, names and images are fairly established ways of performing and reading gender - and thus relevant criteria for our purposes.

${ }^{14}$ The remaining 1 percent has been excluded from the analysis. These were artists that we could not find any information about online.

${ }^{15}$ See e.g. http://mxactivist.tumblr.com/post/69298310891, https://community. spotify.com/t5/Live-Ideas/Make-a-gender-neutral-option-for-profile-signup/idi-p/482938, https://community.spotify.com/t5/Help-Accounts-andSubscriptions/Are-non-binary-people-not-welcome-to-use-Spotify/m-p/1173171/ highlight/true\#M160172, http://mxactivist.tumblr.com/post/69768251170 (all accessed 13/04/2017)

${ }^{16}$ See http://mxactivist.tumblr.com/post/150489712620 (accessed 11/04/2017).

${ }^{17}$ An alternative interpretation could for example be that users who receive outlier recommendations are in much greater need for special musical guidance, and hence are perceived as less independent. Looking at the outlier recommendations, however, many seemed to be artists who are lesser known. This, we argue, indicates that the outlier recommendations are geared towards a more specialized and exploratory taste in music.

\section{References}

Amoore, Louise \& Piotukh Volha (eds.) (2016): Algorithmic Life: Calculative Devices in the Age of Big Data, London and New York: Routledge.

Baker, Paul \& Amanda Potts (2013): “'Why do white people have thin lips?' Google and the perpetuation of stereotypes via auto-complete search forms", Critical Discourse Studies, 10:2, 187-204.

Bannister, Matthew (2006): White boys, white noise: masculinities and 1980s indie guitar rock, London: Ashgate.

Bayton, Mavis (2013): "Women and the electric guitar," Sheila Whiteley (ed.): Sexing the Groove: Popular Music and Gender, New York: Routledge.

Beer, David (2013): Popular Culture and New Media: The Politics of Circulation, London and New York: Palgrave Macmillan.

Berggren, Kalle (2013): "Degrees of Intersectionality: Male Rap Artists in Sweden Negotiating Class, Race and Gender," Culture Unbound, 5, 189-211.

Berry, David M. (2011): The Philosophy of Software: Code and Mediation in the Digital Age, London and New York: Palgrave Macmillan.

Bivens, Rena (2015): "The gender binary will not be deprogrammed: Ten years of coding gender on Facebook," New Media \& Society. Published online ahead of 


\section{Culture Unbound}

Journal of Current Cultural Research

print: http://journals.sagepub.com/doi/abs/10.1177/1461444815621527 (accessed 10/04/2017).

Bivens, Rena \& Oliver L Haimson (2016): "Baking Gender into Social Media Design: How Platforms Shape Categories for Users and Advertisers," Social Media + Society, 2:4, 1-12.

Boshmaf, Yazan, Ildar Muslukhov, Konstantin Beznosov, \& Matei Ripeanu (2011): "The Socialbot Network: When Bots Socialize for Fame and Money," ACSAC, Dec 5-9: http://dl.acm.org/citation.cfm?id=2076746 (accessed 10/04/2017).

Butler, Judith (1990): Gender trouble: feminism and the subversion of identity, New York: Routledge.

Butler, Judith (2004): Undoing Gender. New York: Routledge.

Butler, Judith (2010): "Performative Agency," Journal of Cultural Economy, 3:2, 147-161.

Cheney-Lippold, John (2011): “A New Algorithmic Identity: Soft Biopolitics and the Modulation of Control," Theory, Culture \& Society, 28:6, 164-181.

Cheney-Lippold, John (2017): We are Data: Algorithms and the Making of our Digital Selves, Ne York: NYU Press.

Choi, Grace Y (2016): “'Who Run the Music? Girls!' Examining the Construction of Female Digital Musicians' Online Presence," Popular Music and Society, 1-14, http://dx.doi.org/10.1080/03007766.2016.1174419.

Cohen, Sara (2013): "Men making a scene: rock music and the production of gender," Whiteley, Sheila (ed.): Sexing the Groove: Popular Music and Gender. New York: Routledge.

Dixon-Roman, Ezekiel (2016): "Algo-Ritmo: More-Than-Human Performative Acts and the Racializing Assemblages of Algorithmic Architectures," Cultural Studies Critical Methodologies, 16:5, 482-490.

Drew, Rob (2005): "Mixed Blessings: The Commercial Mix and the Future of Music Aggregation," Popular Music and Society, 28:4, 533-51.

Eriksson, Maria \& Anna Johansson (forthcoming): “"Keep Smiling!': Time, Functionality and Intimacy in Spotify's Featured Playlists," forthcoming in Cultural Analysis, Special Issue on The Inheritance of the Digital: Ethnographic Approaches to Everyday Realities In, Of, And Through Digital Technologies.

Featherstone, Mike (2006): "Archive," Theory, Culture \& Society, 23:2-3, 591-96.

Frith, Simon \& Angela McRobbie (1991): "Rock and sexuality," Frith, Simon \& Andrew Goodwin (eds.): On Record: Rock, pop and the written word. London/New York: Routledge, 371-389.

Fuller, Matthew (2003): Behind the blip. Essays on the culture of software, Brooklyn, NY: Autonomedia.

Gadir, Tami (2016): "Resistance or Reiteration? Rethinking Gender in DJ Cultures," Contemporary Music Review, 35:1, 115-129.

Galuszka, Patryk (2015): "Music Aggregators and Intermediation of the Digital Music Market," International Journal of Communication, 9, 254-73.

Gavanas, Anna \& Rosa Reitsamer (2013): "DJ technologies, social networks and gendered trajectories in European DJ cultures", Attias, Bernardo, Anna Gavanas \& Hillegonda C Rietweld (eds.): DJ culture in the mix: Power, technology, and social change in electronic dance music, New York: Bloomsbury Academic, 51-77.

Gillespie Tarleton (2014) "The Relevance of Algorithms", Tarleton Gillespie, Pablo J. Boczkowski \& Kirsten A. Foot (eds.) Media Technologies: Essays on Communication, Materiality, and Society, Cambridge \& London: MIT Press, 167-194.

Haraway, Donna (1997): Modest_Witness@Second_Millennium.FemaleMan (C_Meets OncoMouse: Feminism and Technoscience. New York: Routledge.

Hayles, Katherine (1999): How We Became Posthuman: Virtual Bodies in Cybernetics, Literature and Informatics. Chicago: University of Chicago Press.

Introna, Lucas D \& Helen Nissenbaum (2000): "Shaping the Web: Why the Politics of 


\section{Culture Unbound}

Journal of Current Cultural Research

Search Engines Matters," The Information Society, 16:3, 169-185.

Jeffries, Michael P (2011): Thug life: race, gender, and the meaning of hip-hop, Chicago: Chicago University Press.

Kitchin, Rob (2017): “Thinking Critically about and Researching Algorithms," Information, Communication \& Society, 20:1, 14-29.

Kjus, Yngvar (2016): "Musical exploration via streaming services: The Norwegian experience," Popular Communication, 14:3, 127-136.

Lamere, Paul (2014): “Gender Specific Listening," Music Machinery, Feb 10: https:// musicmachinery.com/2014/02/10/gender-specific-listening/ (accessed 10/04/2017)

Leonard, Marion (2007): Gender in the Music Industry: Rock, Discourse and Girl Power, Aldershot, England: Ashgate.

Maasø, Arnt (2017): "Music Streaming, Festivals, and the Eventization of Music," Popular Music, 1-22, https://doi.org/10.1080/03007766.2016.1231001.

Mackenzie, Adrian (2006): Cutting Code: Software and Sociality, New York: Peter Lang.

Mager, Astrid (2012): “Algorithmic Ideology," Information, Communication \& Society, 15:5, 769-787.

Manovich, Lev (2013): Software Takes Command, New York: Bloomsbury.

Modell, Amanda (2015): "Mapping the Music Genome: Imaginative Geography in Pandora Internet Radio and the Genographic Project," Media Fields Journal, 10, 2-13.

Morris, Jeremy W (2012): "Making Music Behave: Metadata and the Digital Music Commodity," New Media \& Society, 14:5, 850-66.

Morris, Jeremy W (2015): Selling digital music, formatting culture, Oakland, California: University of California Press.

Nakamura, Lisa (2002): Cybertypes: race, ethnicity, and identity on the Internet, New York: Routledge

Nylund Hagen, Anja (2015): "The Playlist Experience: Personal Playlists in Music Streaming Services, Popular Music and Society, 38:5, 625-645.

Olofsson, Jennie (2015): “'Did you mean: why are women cranky?': Google - a means of inscription, a means of de-scription?," in Svensson Patrick \& David Theo Goldberg (eds.), Between humanities and the Digital, Cambridge, MA: MIT Press.

Parisi, Luciana (2013): Contagious architecture: computation, aesthetics, and space, Cambridge, MA: MIT Press.

Pickering, Andrew (2011): The Cybernetic Brain: Sketches of Another Future, Chicago \& London: University of Chicago Press.

Pough, Gwendolyn D (2004): Check It While I Wreck It: Black Womanhood, Hip-Hop Culture, and the Public Sphere, Boston: Northeastern University Press.

Railton, Diane (2001): “The gendered carnival of pop," Popular Music, 20:3, 321-331.

Rogers, Richard (2013): Digital methods, Cambridge, MA: The MIT Press.

Ruppert, Evelyn, John Law \& Mike Savage (2013): "Reassembling Social Science Methods: The Challenge of Digital Devices," Theory, Culture \& Society, 30:4, 22-46.

Seaver, Nick (2014): “On Reverse Engineering: Looking for the Cultural Work of Engineers," The Atlantic, Jan 28: https://medium.com/anthropology-and-algorithms/ on-reverse-engineering-d9f5bae8712 (accessed 10/04/2017).

Seaver, Nick (2013): "Knowing Algorithms," Media in Transition 8, Cambridge, MA: http://nickseaver.net/papers/seaverMiT8.pdf (accessed 13/04/2017).

Straw, Will (2013): "Sizing up record collections: gender and connoisseurship in rock music culture," Sheila Whiteley (ed.): Sexing the Groove: Popular Music and Gender, New York: Routledge.

Sundén, Jenny (2015): "On trans-, glitch, and gender as machinery of failure," First Monday, 20:4: https://doi.org/10.5210/fm.v20i4.5895 (accessed 13/04/2017)

Thrift, Nigel (2008): Non-Representational Theory: Space, Politics, Affect, London \& New York: Routledge. 
Wajcman, Judy (2007): "From Women and Technology to Gendered Technoscience," Information, Communication \& Society, 10:3, 287-298.

Werner, Ann \& Sofia Johansson (2016): "Experts, dads and technology: Gendered talk about online music,"International Journal of Cultural Studies, 19:2, 177-192. Whiteley, Sheila (ed.) (2013): Sexing the Groove: Popular Music and Gender, New York: Routledge.

Whiteley, Sheila \& Jennifer Rycenga (eds.) (2006): Queering the popular pitch, New York: Routledge. 